\title{
Anticipation of Social Interaction Changes Implicit Approach-Avoidance Behavior of Socially Anxious Individuals
}

\author{
M. J. Voncken • M. Rinck • A. Deckers • \\ W.-G. Lange
}

Published online: 30 October 2011

(c) The Author(s) 2011. This article is published with open access at Springerlink.com

\begin{abstract}
Earlier research has revealed implicit avoidance of social stimuli in social anxiety (SA). This study investigated such reactions in anticipation of social interaction. High $(n=24)$ and low $(n=22)$ SA females were assessed in anticipation of a getting-acquainted conversation (anticipation) and in a no-conversation-expected (neutral) condition. The Face-Turn Approach Avoidance Task was used in which participants responded to profiles of human faces or control stimuli by either pulling (approach) or pushing (avoidance) a joystick. Upon pulling, the stimuli turned toward the participant, while they turned away upon pushing. The results demonstrated the expected decreased approach response to faces in the neutral condition for the high SAs compared to the low SAs group. Unexpectedly, in the anticipation condition the high SAs showed increased approach tendencies to faces whereas, the low SAs demonstrated a decreased approach response. The implicit social approach response of the high SAs in the anticipation condition is discussed.
\end{abstract}

Keywords Social anxiety - Implicit behavior . Approach behavior . Avoidance behavior

M. J. Voncken $(\bowtie) \cdot$ A. Deckers

Department of Clinical Psychological Science, Faculty of Psychology and Neuroscience, Maastricht University, P.O. Box 616, 6200 MD Maastricht, The Netherlands e-mail: m.voncken@maastrichtuniversity.nl

M. Rinck - W.-G. Lange

Behavioural Science Institute, Radboud University Nijmegen,

Nijmegen, The Netherlands

\section{Introduction}

Patients with social anxiety disorder (SAD) excessively fear being negatively evaluated by others (APA 2001; Bögels et al. 2010). These patients tend to avoid social interaction altogether or show subtle avoidance responses during social interaction. A line of studies shows that some of these avoidance behaviors in SAD consist of strategic, so called safety behaviors, such as restrictive behaviors (e.g., remaining silent in order to not say anything stupid), pro-active behaviors (e.g., making excuses for one's supposedly despicable appearance), and concealing physical symptoms of anxiety (e.g., wear clothes that conceal sweating; Cuming et al. 2009). Safety behaviors are perceived as being under high voluntary control, and although socially anxious individuals (SAs) regard such strategies as helpful, these behaviors are counterproductive and undermine social interaction (McManus et al. 2008). It is assumed that the use of safety behaviors may explain why SAs are perceived as less likeable than people without social anxiety as evidenced by a body of studies (e.g., Alden and Wallace 1995; Creed and Funder 1998; Meleshko and Alden 1993; Voncken et al. 2008, 2010). Such a true negative evaluation of SAs is thought to fuel their fear of negative evaluation (Alden 2001). Indeed, current cognitive models of SAD state that safety behaviors play an essential role in the maintenance of SAD (Clark 2001; Hofmann 2007; Rapee and Heimberg 1997). In fact, a number of studies demonstrate that dropping such strategic safety behaviors seems essential to change social anxiety symptoms in SAD (Furukawa et al. 2009; Kim 2005; McManus et al. 2009; Taylor and Alden 2010; Wells et al. 1995).

In sum, there is accumulating evidence for the essential role of strategic safety behaviors in the maintenance of 
SAD. However, part of the avoidance repertoire of SAs may not be strategic but rather automatic, on an implicit, unconscious level. Indeed, Foa and Kozak (1985) put forward that not all elements of fear are accessible for introspection. For instance, implicit avoidance tendencies in social anxiety could involve subtle avoidance of eye-contact or subtly keeping one's distance. Indeed, Rinck et al. (2010), using immersive virtual environments, showed that with an increasing degree of social anxiety, participants became slower in approaching computer-generated persons (avatars) and kept more distance from these avatars. Similarly, Vrijsen et al. (2010) showed that SAs show another positive social behavior less frequently, namely, automatic mimicry (of, e.g., head movements) of their interaction partner. This way, automatic behaviors, just like strategic safety behaviors, might deteriorate the quality of a social interaction and elicit a truly more negative evaluation by others, which in turn maintains social anxiety.

The Approach-Avoidance Task (AAT) developed by Rinck and Becker (2007) is another measure to assess implicit avoidance behavior. It is based on the idea that human beings have a tendency to automatically approach pleasant stimuli while avoiding unpleasant or threatening ones (Chen and Bargh 1999; Solarz 1960). Thus, inevitable automatic evaluation of a stimulus is thought to influence subsequent behavior, even when stimulus valence is not task-relevant (Lange et al. 2008). In a common version of the AAT, participants are placed in front of a computer screen and a joystick, and are asked to categorize pictures according to irrelevant attributes such as background color or format, either by pulling the joystick towards themselves (approach) or by pushing the joystick away (avoidance). When the joystick is pulled, the displays increase in size, as if approached, and decrease in size when being pushed. Speeded pushing and slowed pulling are interpreted as "avoidance" of a stimulus, while speeded pulling and slowed pushing reflect "approach".

The AAT has shown to be a useful tool to investigate implicit avoidance behavior in different anxious populations (Rinck and Becker 2007) and first evidence is available for implicit avoidance tendencies of social stimuli in SAs. Heuer et al. (2007) found that SAs pushed (avoided) emotional faces (angry as well as happy faces) more quickly than they pulled (approached) these faces, when compared to non-anxious control participants (NACs) and neutral control faces. In line with this finding, Lange et al. (2008) showed that SAs, when compared to NACs, were faster in pushing than pulling pictures of facial crowds that consisted of an increasing number of angry faces. In addition, happy crowds were avoided as well. Moreover, Roelofs et al. (2010) found the same results in response to angry and happy faces with a different version of the AAT. Here, participants did not pull or push a joystick, but responded with comparable arm-flexion to press an upper button or arm-extensions to press a lower button placed on a vertical column. Participants were not asked to respond to e.g., background colors of the stimuli but were directly instructed to push the upper button for positive faces and the lower button for negative faces (or vice versa).

While these studies demonstrated implicit avoidance tendencies in SAs in a context that is rather non-social and thus, non-threatening, research is scarce about whether these tendencies also occur in actual social/threatening situations, as safety behaviors would do. However, two studies indicate that implicit avoidance tendencies of SAs in a neutral context increase during actual social stress. These studies investigated implicit avoidance tendencies at baseline and right after a socially stressful situation (Trier Social Stress Task; Roelofs et al. 2005, 2009). With their version of an AAT, they showed that SAs, compared to participants without SA, were faster in avoiding than approaching emotional faces in the social stress condition compared to baseline. However, one main purpose of safety or avoidance behaviors in SA is to prevent social stress from occurring and, therefore, should be most frequent in anticipation or during social stress and not after a social task. Moreover, in daily life SAs are mostly confronted with social interaction stress instead of stress caused by giving speeches or extreme performance evaluations as in the Trier Social Stress Test. Finally, behavioral problems in SAD are more prominent in conversations than in speech situations (Voncken and Bögels 2008). Therefore, the present study will investigate implicit avoidance tendencies of SAs in anticipation of an upcoming conversation.

The aim of the present study was to assess implicit approach and avoidance behavior under neutral conditions and in anticipation of an upcoming conversation with an unknown person in high and low SAs. Accordingly, we developed a variation of the AAT, the Face-Turn AAT, in which we aimed to simulate a situation in which the participants took the initiative to make contact with the model on the screen by pulling the joystick (approach), or to avoid such contact by pushing the joystick (avoidance). Therefore, participants were presented with a profile of a neutral face or a neutral control stimulus that turned toward the participant upon pulling the joystick and turned away upon pushing the joystick. Moreover, we informed the participants that they would meet one of the individuals presented in the Face-Turn AAT in the upcoming social interaction. To investigate whether responses on the Face-Turn AAT were related to an explicit measure of the wish to make contact with the models on the screen, the participants rated the models on the Desire for Future Interaction scale (DFI). In a within-subjects design, for each participant the Face-Turn AAT was administered on two assessment 
moments, 2 weeks apart. In one session, participants were informed that they would participate in a getting-acquainted interaction after completion of the AAT (anticipation condition), and in the other session not (neutral condition). The order of the two test-sessions was counterbalanced. For reasons of convenience and homogeneity of the sample, it was chosen to only include females.

It was expected that, (I) in the neutral condition, high SAs would be faster (Ia) in turning the faces away from them (avoidance) and (Ib) slower in turning the faces towards them (approach) when compared to the control stimuli and the low SAs. In addition, (II) when anticipating a social interaction, we expected these effects to be more pronounced. Last, it was investigated whether (III) the responses on the Face-Turn AAT were related to explicit measures of the wish to make contact with the models on the screen under each condition for each group.

\section{Method}

\section{Participants}

During a pre-screening, 229 first year female students of psychology and health sciences from Maastricht University filled out the Social Interaction Anxiety Scale (SIAS; Mattick and Clarke 1998). First, students $(n=1)$ who scored 1.5 SD above the mean of the SIAS of patients with SAD (as reported by Mattick and Clarke 1998: mean SIAS: 34.6; $\mathrm{SD}=16.4$ : e.g., equal or above 60 ), and students $(n=9)$ who scored 1.5 SD below the mean of the SIAS in the current study (e.g., equal or lower than 6 on the SIAS; mean SIAS $=23.6, \mathrm{SD}=12.3$ ), were excluded as these may be a deviant levels of social anxiety. To compose the high SAs group, the top $25 \%$ of the individuals with the highest score ( $n=60$, range SIAS 30-60) were invited for participation, and $25 \%$ of the students with the lowest scores $(n=58$, range SIAS 7-15) were invited.

The selected individuals $(n=118)$ were invited for two assessments (neutral and anticipation condition). Of the 61 individuals who participated, 15 had to be excluded: three participants did not attend both assessment points, and of 2 individuals some of the data were lost due to technical failure. Because the pre-screening of the SIAS occurred during a mass screening several months before the experiment, we carefully checked whether the selected participants could still be considered high or low SAs. Therefore, the participants filled-out the SIAS again at the two assessment points of our study, and with a median spilt we divided our sample again into a high and low SAs group for these two assessment points. The final groups were composed only of individuals who consistently, at both assessment points, were considered to be either high or low
SA. This resulted in the exclusion of another 10 participants. After all exclusions, 22 high SAs and 24 low SAs were included in the analyses.

\section{Questionnaires}

\section{Social Anxiety and Depression}

The SIAS (Mattick and Clarke 1998) consists of 20 items of self-statements describing one's typical cognitive, affective, or behavioral reactions to situations that involve social interaction in dyads or groups. These are rated from 0 (not at all characteristic or true of me) to 4 (extremely characteristic or true of me). The SIAS has sound psychometric properties (Mattick and Clarke 1998). The participants also completed the anxiety subscale of the Liebowitz Social Anxiety Scale (LSAS; Liebowitz 1987) and the Center for Epidemiologic Studies Depression Scale (CES-D; Radloff 1977).

\section{STAI-State}

To assess changes in state-anxiety level at different points throughout the experiment, the participants filled out the Dutch state version of the State-Trait Anxiety Inventory (STAI-state: Van der Ploeg et al. 1980) right before the Face-Turn AAT. The STAI-state consists of 20 self-statements that are rated on a 4-point scale.

\section{Face-Turn AAT}

\section{Stimuli}

In order to create the effect of the stimuli turning toward and away from the participant, seven pictures each were taken of the faces of 9 male and 9 female models, and a computer monitor as control stimulus. Seven photos were taken of each individual, from frontal view in steps of $30^{\circ}$ via a profile view to a picture of the back of the individual's head (see Fig. 1). The models were instructed to look neutral but slightly friendly. In a pilot test, 15 other female students (age range 20-25) rated the pictures indeed as looking slightly friendly (on a 7 point Likert scale: $\mathrm{M}=5.0 ; \mathrm{SD}=.3$; range 3.9-5.4) and having a slightly positive emotional expression (on a 7 point Likert scale: $\mathrm{M}=4.8 ; \mathrm{SD}=.3$; range 4.3-5.5). Seven pictures each were also taken of nine identical computer monitors. To give these control stimuli an individualistic look, each of the depicted computer monitors showed a different landscape.

\section{Apparatus and Procedure}

A joystick type Logitech Attack 3 was positioned about halfway $(25 \mathrm{~cm})$ between the participant and the computer 
a

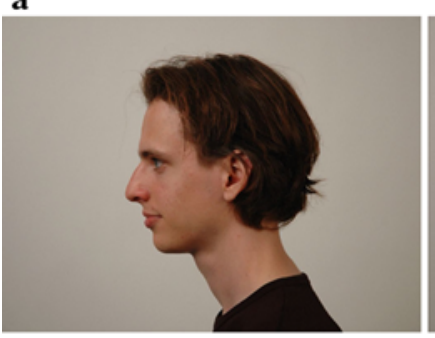

b
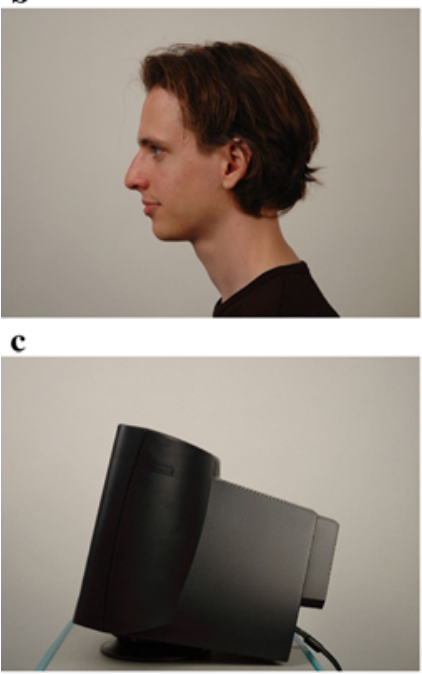

d
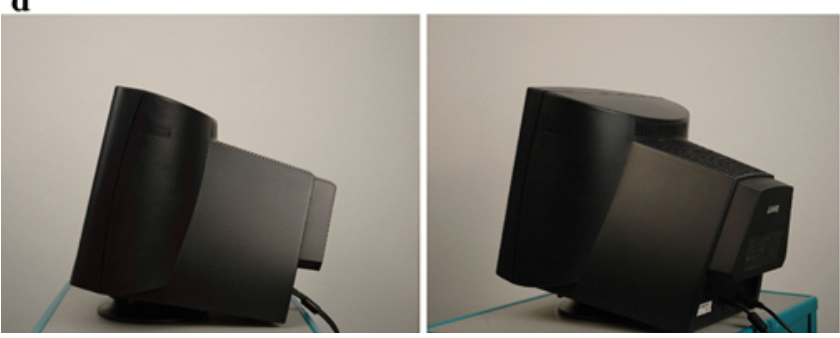

Fig. 1 Pictures of the stimuli in the sequence in which they were presented to the participants: starting with the profile pictures, in steps of $30^{\circ}$ to either frontal view or back view. a Sequence of the pull

screen, tightly attached to the table. Each trial was selfpaced: participants had to press the fire-button while the joystick was in the resting position and the screen was blank. After pressing the button, either a profile 'face' or 'computer monitor' stimulus appeared in the center of the screen. Participants were instructed to respond to the direction to which the face or computer monitor pointed, either left or right. Half of the participants were instructed to push the left-directed stimuli and to pull the rightdirected stimuli, and for the other half of the participants these instructions were reversed.

By pushing the joystick, the stimulus face or computer monitor turned away from the participant in steps of $30^{\circ}$. Here the last picture showed the backside of the person's head or the computer monitor. By pulling the joystick, the face or computer monitor turned toward the participant in
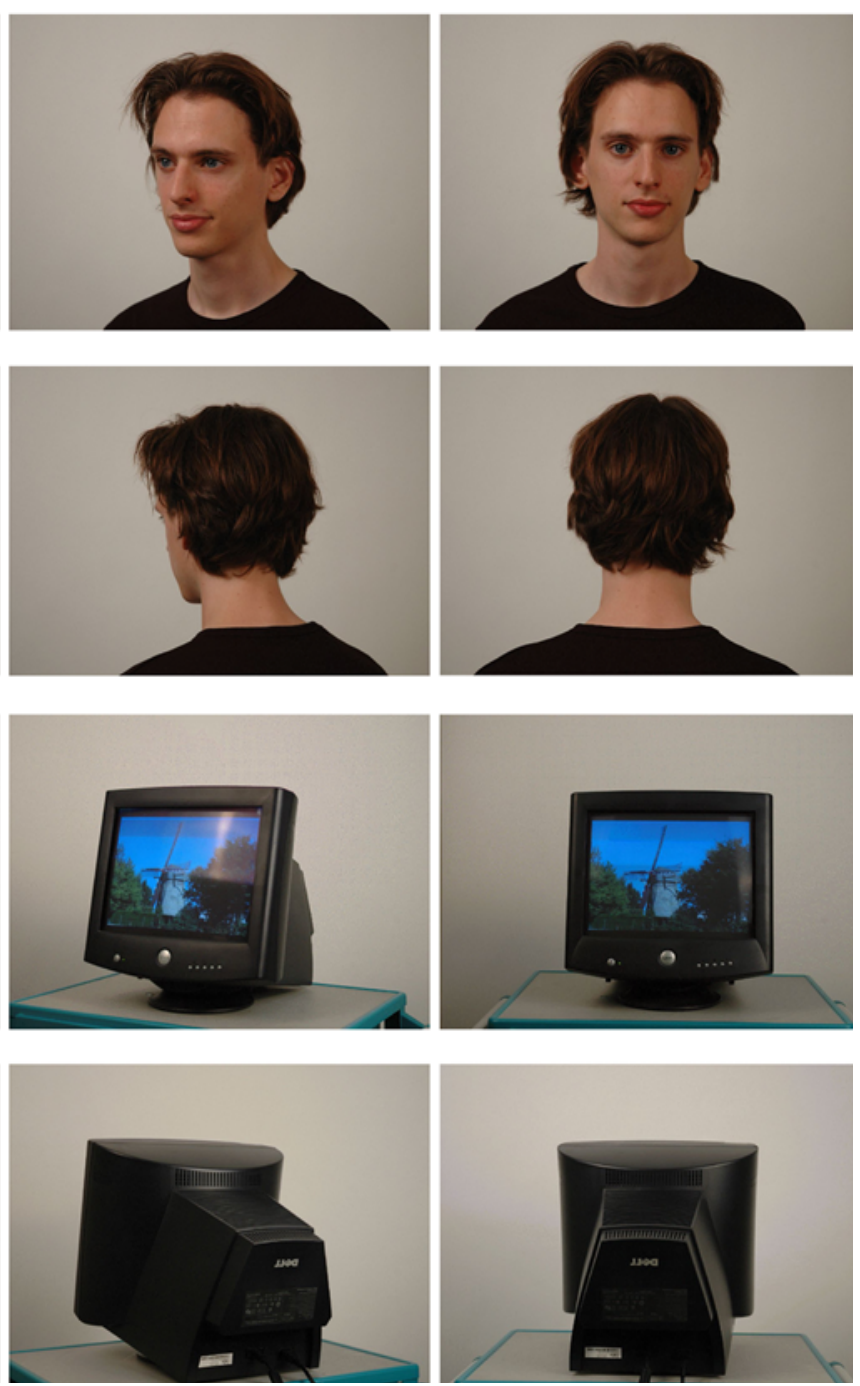

response for a male face. $\mathbf{b}$ Sequence of the push response for a male face. c Sequence of the pull response for a computer monitor. d Sequence of the push response for a computer monitor

steps of $30^{\circ}$. In this instance the last picture showed the face of the person or the front of the computer monitor. As soon as the stimulus face or computer monitor was completely turned and the joystick was pushed or pulled all the way, the stimulus disappeared from the screen. The participants were given three practice trials (a male and a female face and a computer monitor) to get acquainted with the task.

Two versions of each actor/monitor, one facing left and one facing right, were used. In total, each participant completed one practice trial for each stimulus (male, female, computer monitor) and subsequently five blocks of 48 trials ( 8 males, 8 females, 8 computer monitors not used in the practice trials) resulting in 240 trials. The order of the trials was pre-randomized, and participants had a short break after 120 trials. The reaction time and the accuracy of the responses were measured. 


\section{Explicit Wish to Make Contact}

Participants rated the profile version of the AAT faces with a computerized version of the DFI (Coyne 1976), a measure of wish to engage in future interaction (Boswell and Murray 1981; Papsdorf and Alden 1998; Winer et al. 1981). Due to time constraints, each participant rated each picture on a 5-point Likert scale with respect to a random selection of 4 items from the 8 original items by mouseclick. In the current study, the internal consistency of the DFI was high (Cronbach's $\alpha$ : ranged from .81 to .91).

\section{Procedure}

All participants were assessed twice, with 2 weeks in between. One assessment point was the 'neutral' condition, while the other one was the 'anticipation of social interaction' condition (anticipation condition). The order of these conditions was counterbalanced. Before testing, the participants were informed which one of the two assessments involved a social interaction.

\section{Neutral Condition}

Upon arrival in the laboratory, the participants were informed that this assessment only consisted of filling out questionnaires and completing a computer task.

\section{Anticipation of Social Interaction Condition}

Upon arrival in the laboratory, the participant was informed that she was to hold a conversation after completing questionnaires and a computer task. The experimenter showed the participant the area where the conversation would be held. Here two chairs and a camera were placed. The experimenter took place in one chair and while pointing to the camera said: "This is the camera. After the computer task you will need to take place on this chair. Please take a seat now. The other person will sit in my chair. The person with whom you will have a conversation will be depicted in the computer task. With regard to the social task, it is important that you do not move the chair, otherwise it will not be recorded well. The purpose of the conversation is to get to know each other. It is important is that you are the one that starts the conversation and keeps it going. The camera will record the conversation. Video observers will evaluate your social skills later on."

After either the neutral or the anticipation of social interaction instructions, the participants were seated in an area where a computer, a joystick, and a package of questionnaires were prepared. Here, they first filled out the STAI, conducted the Face-Turn AAT and rated the faces on likeability on the computer. Then they completed the set of questionnaires (i.e., SIAS, LSAS and CES-D). In the anticipation condition, the participants were seated in a waiting room for $5 \mathrm{~min}$ and then conducted the conversation with a confederate. These data were used for another study that will be reported elsewhere (Voncken and Dijk, under review). After each assessment, they were thanked for their effort and paid, and after the final assessment they were debriefed.

\section{Results}

Participants' Characteristics

As expected, in both conditions, the high SAs group had significantly higher ratings on the SIAS, the anxiety subscale of the LSAS, and the CES-D than the low SAs group (see Table 1). Further, a repeated-measures ANOVA with time of assessment (neutral vs. anticipation condition) as within-subjects variable and group (high vs. low SAs) as between-subjects variable was used to investigate stateanxiety differences between the two assessments as measured with the STAI-state. The participants showed a non-significant trend towards having higher state anxiety in the anticipation when compared to the neutral condition, $F(1,44)=3.4, P<.07$, partial $\eta^{2}=.07$. Moreover, a main effect of group indicated that the high SAs group had a higher STAI-state rating across both conditions than the low SAs group $F(1,44)=12.9, P<.005$, partial $\eta^{2}=.23$. No interaction effect occurred, $F(1,44)=2.2, n s$, partial $\eta^{2}=.05$. As we had an a-priory hypothesis that the high SAs group would specifically be reactive to our manipulation, we tested this with a paired $t$ test. Indeed, the high SAs group showed in increase on the STAI from the neutral to the anticipation condition, $t(23)=2.1, P<.05$ (see Table 2).

\section{Face-Turn AAT}

AAT-effects are usually calculated by computing a difference score between reaction times for pull and push movements. Recently, it has been suggested, however, that approach and avoidance may be separate entities. For instance, in research of craving for alcohol and food, it is assumed that both action tendencies can exist relatively independently of each other (see Cartwright and Stritzke 2008; Stritzke et al. 2007). Moreover, a recent study in obsessive-compulsive disorder found differences between high and low contamination fear groups only for the approach, but not for participants' avoidance responses (Najmi et al. 2010). In order to clarify whether high SAs are characterized by faster avoidance (push) or slower approach responses (pull), both movements were analyzed separately. 
Table 1 Means and standard deviations of the high social anxiety (high SAs) and the low social anxiety (low SAs) group on social anxiety and depression measures assessed at the two assessment points

\footnotetext{
$* P<.001$, two-tailed

a Social Interaction anxiety scale

b Liebowitz social anxiety scale

c Centre for epidemiologic studies depression scale
}

\begin{tabular}{lll}
\hline & $\begin{array}{l}\text { Low SAs group } \\
n=22 \\
\text { mean (SD) }\end{array}$ & $\begin{array}{l}\text { High SAs group } \\
n=24 \\
\text { mean (SD) }\end{array}$ \\
\hline SIAS $^{\mathrm{a}}$ & $12.2(4.3)$ & $38.2(11.0)^{*}$ \\
$\begin{array}{l}\text { Neutral condition } \\
\text { Anticipation for social interaction }\end{array}$ & $15.2(4.9)$ & $38.1(11.6)^{*}$ \\
LSAS ${ }^{\mathrm{b}}$, anxiety subscale & $14.8(5.5)$ & $32.6(9.9)^{*}$ \\
Neutral condition & $17.6(10.9)$ & $32.3(10.9)^{*}$ \\
Anticipation for social interaction & & $18.8(12.8)^{*}$ \\
CES-D & $7.8(6.1)$ & $18.2(11.7)^{*}$ \\
Neutral condition & $7.5(6.0)$ & \\
Anticipation for social interaction & & \\
\hline
\end{tabular}

Table 2 Means and standard deviations of the high SAs and the low SAs group on the STAI-state right before the face-turn AAT during the neutral and anticipation for social interaction condition

\begin{tabular}{llr}
\hline & $\begin{array}{l}\text { Low SAs group } \\
(n=22) \\
\text { mean (SD) }\end{array}$ & $\begin{array}{l}\text { High SAs group } \\
(n=24) \\
\text { mean (SD) }\end{array}$ \\
\hline Neutral condition & $33.8(6.8)^{\mathrm{a}}$ & $38.5(7.8)^{\mathrm{b}}$ \\
Anticipation condition & $34.2(5.3)^{\mathrm{a}}$ & $42.0(7.3)^{\mathrm{c}}$ \\
\hline
\end{tabular}

Means with different superscripts differ significantly, $P<.05$, two-tailed

Note that the overall interaction between group and condition was not significant

In the current study, the computer monitors were used as control stimuli for the human faces. Therefore, a difference score was calculated by subtracting the reaction time for human faces from reaction time for computer monitors. For the push movements, the higher this difference score, the more avoidance the participants showed toward the faces compared to computer monitors. For the pull movements, the higher this difference score, the more approach the participants showed toward the faces compared to computer monitors. First, we investigated whether the order of the assessment moments (neutral condition first vs. anticipation condition first), despite our counterbalancing, had any effect. No effects of order appeared in the planned repeated-measures ANOVAs [Avoidance: $F(1,42)=.4, n s$, partial $\eta^{2}=.00$; Approach: $F(1,44)=.0, n s$, partial $\left.\eta^{2}=.00\right]$.

\section{Avoidance}

The 2 (condition: neutral, anticipation) $\times 2$ (group: high, low SAs) repeated-measures ANOVA did not show the expected two-way interaction, $F(1,44)=.6, n s$, partial $\eta^{2}=.00$. No effect for condition, $F(1,44)=.6, n s$, $\eta^{2}=.01$, or group $F(1,44)=1.4, n s$, partial $\eta^{2}=.03$, occurred either.

\section{Approach}

The 2 (condition: neutral, anticipation) $\times 2$ (group: high, low SAs) repeated-measures ANOVA showed a significant two-way interaction, $F(1,44)=10.5, P<.005$, partial $\eta^{2}=.19$ (Fig. 2). No effect for condition, $F(1,44)=.3, n s$, partial $\eta^{2}=.01$, or group $F(1,44)=.1, n s$, partial $\eta^{2}=.00$, occurred. For the neutral condition, the high compared to the low SAs group showed the expected decreased approach response to human faces compared to computer monitors, $t(44)=2.4, P<.02$. Unexpectedly, this decreased approach response of the high SAs group reversed in the anticipation condition to an increased approach response to human faces, $t(23)=2.3, P<.05$. The low SAs showed the exact opposite pattern, the increased approach in the neutral condition reversed to an decreased approach to human faces in the anticipation condition, $t(21)=2.6, P<.05$. In the anticipation condition, the difference between the high and low SAs group was approaching significance, $t(44)=1.8, P<.10$.

\section{Explicit Wish to Make Contact}

No significant correlations appeared between the approach or avoidance responses of the Face-Turn AAT and the explicit wish to make contact with the models of the FaceTurn AAT in the groups, neither for the neutral (i.e., avoidance: high SAs: $r=.17, n s$; low SAs $r=.07, n s$; approach: high SAs: $r=.22$; low SAs: $r=-.13, n s$ ) nor for the anticipation condition (i.e., avoidance: high SAs: $r=.28, n s$; low SAs $r=.06$; approach: high SAs: $r=.31, n s ;$ low SAs: $r=-.04, n s)$. 


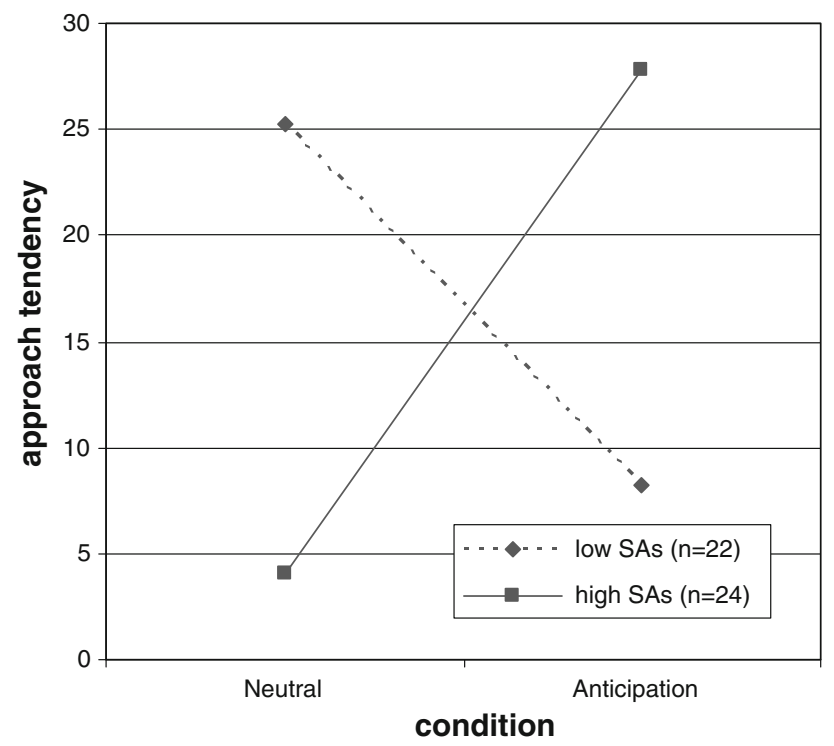

Fig. 2 Difference scores of the reaction time pulling computer monitors minus faces, during the neutral and anticipation condition for high and low SAs. A higher difference score indicates an increased approach tendency toward faces compared to computer monitors, a lower score indicates a decreased approach tendency toward faces compared to computer monitors

\section{Discussion}

This study investigated implicit approach and avoidance tendencies of high and low SAs during neutral circumstances and in anticipation of an upcoming social interaction. A new version of the AAT (Face-Turn AAT) was developed in which human faces and control stimuli (computer monitors) turned toward the participants on pull (approach) and away on push (avoidance) responses. No differences between the groups emerged with regard to the avoidance tendencies. However, as expected, under neutral conditions, high SAs compared to low SAs showed decreased approach tendencies to faces. Unexpectedly, high SAs showed increased approach tendencies to human stimuli in anticipation of a social interaction, whereas, in the low SAs, when anticipating a social interaction the increased approach reversed to a decreased approach. These results should be seen as tentative as our manipulation did not result in the expected group $\times$ condition interaction on anxiety level (STAI). However, the a priori analysis for specifically the high SA group did show that the anxiety level increased in this group.

First, the results for the low SAs will be discussed. The low SAs showed the expected pattern of increased approach toward human faces under neutral conditions. This seems to reflect the evolutionary tendency of human beings to favor human stimuli over non-human stimuli (for an overview, see Nelson et al. 2005). Anticipating a social interaction, this approach tendency reversed into a decreased approach for human stimuli. This may reflect implicit avoidance behavior in anticipation of social stress. Interestingly, this contradicts their subjective reports because the low SAs did not report increased state anxiety in the anticipation compared to the neutral condition.

With regard to the findings for the high SAs group, in line with previous studies (Heuer et al. 2007; Lange et al. 2008; Roelofs et al. 2010), it can be concluded that under neutral circumstances, this group showed implicit avoidance of human faces, relative to non-human stimuli when compared to the low SAs group. In more detail, our study shows that this avoidance response was not characterized by a faster implicit avoidance response but by a slower approach response. Technical aspects of the Face-Turn AAT may explain why no differences between the groups were found for the avoidance response. The stimuli in the push condition were always turning away from the participants and, thus, for the stimulus faces mainly the back of the head was visible. This might have made these stimuli less socially relevant and, therefore, pushing did not elicit differences in response times between the two groups. Moreover, floor effects are more common for push responses than for pull responses. However, our finding might also indicate that SA is mainly characterized by slower approach and not so much by increased avoidance. This is in line with the findings in a recent study on contamination fear (Najmi et al. 2010). In their study, high fearfuls did not differ from the low fearfuls on pushing (avoidance) but only on pulling (approach) responses for their fear-related stimuli. Moreover, Rinck et al. (2010) using virtual reality tools, showed that the more socially anxious participants were slower in approaching computergenerated persons (avatars), but not faster when going back to the starting point, and that they kept more distance from these avatars. Indeed, a substantial part of strategic safety behaviors in SAs consists of a decreased approach response such as restrictive behaviors (e.g., remaining silent, not talking to certain people) or concealing anxiety symptoms (Cuming et al. 2009). Although, evidently more research is needed, SA might be characterized primarily by problematic approach behavior instead of avoidance behavior. If so, treatment should focus on actively approaching and making contact with others and not only on dropping safety or avoidance behavior.

The finding that high SAs showed approach tendencies for human faces in anticipation of social contact was unexpected. It needs to be noted here that although the high SAs group did show an increase in anxiety from the neutral to the anticipation situation, the overall interaction between group and assessment moment was not significant. Therefore, this result needs replication and is only tentative. Moreover, this finding seems to contradict those of previous studies (Roelofs et al. 2005, 2009). These studies found 
that the avoidance tendencies of SAs at baseline amplified in response to a high social stressor (a speech) instead of changing into an approach tendency. However, this may be due to procedural differences between the studies. That is, Roelofs' participants were assessed right after a highly stressful social situation, rather than in anticipation of it. Although, the participants were more stressed at that moment than during the neutral assessment, the importance and emotional relevance of the social stimuli might not have been apparent anymore as participant did not need to interact with others after the assessment. Moreover, in our study the human faces turned toward the participants and participants were told that they would meet one of the persons displayed in the task, whereas, in the Roelofs' studies, the human faces were shown frontally, did not turn and there was no connection between the faces and the social interaction. Therefore, the human stimuli in our study should have been of more importance and emotional relevance for high SAs.

The approach tendencies of the high SAs group in our study give room for two speculative interpretations. First, it seems plausible that the high SAs in anticipation of the social interaction expected that they would not be able to avoid the upcoming social interaction, and that this increased the importance and emotional significance of the human stimuli. High SAs may be more motivated in the anticipation condition to see the frontal picture of the face in order to gain more information about the person that they cannot avoid meeting in a few minutes. This could explain why they were faster in turning the human pictures towards them than the computer monitors. In the same line, Mackintosh and Mathews (2003) postulate that when the importance and emotional significance of threatening stimuli increase, anxiety patients show a reflexive attentional bias toward threat instead of avoidance of threat.

A second, contrasting and more speculative interpretation of the approach tendency of the high SAs when anticipating social interaction would be that this reflects their implicit eagerness to have contact with others. It may be that only under circumstances in which they are confronted with an unavoidable social interaction, as in the anticipation condition, their wish to make contact with others becomes apparent. The diagnosis of SAD implicitly assumes that, in contrast to, e.g., schizoid personality disorder, their desire for social interaction is still intact. Taking into consideration that SAD patients report severe problems in developing satisfying relations (see Alden and Taylor 2004, 2010, for reviews) they may well have an increased implicit wish to make contact with others. Indeed, Davila and Beck (2002) showed that SAs have a dependent interpersonal style in close relationships, suggesting that they rely excessively on close others to compensate for their problems in social contact. Moreover, in the study by Kashdan and Wenzel (2005), dyads of high and low SAs participated in conversations of $45 \mathrm{~min}$ of personal disclosure. Afterwards, the SAs dyads appreciated these social interactions more than the low SAs or mixed high and low SAs dyads. The authors interpreted this finding to suggest that, due to their avoidance, social activities are rare, but highly desirable for high SAs. It may be that such a deprivation from social interactions results in an eagerness to have satisfying interactions with others. This eagerness for social interactions may then become apparent at an implicit level when the high SAs are confronted with an upcoming, unavoidable social interaction, as in our study. Of course, this interpretation is speculative and needs more study.

A number of limitations need to be addressed. First of all, how implicit avoidance behavior in SA relates to actual behavior is still unclear. Although, Rinck and Becker (2007) demonstrated the link between spider-related avoidance behavior in the AAT and avoidance of real spiders by spider fearful individuals, no study so far has investigated this link in SA. Moreover, the current study itself also has a number of limitations. First, the test-retest reliability of the Face-Turn AAT is not established, yet. Second, it is not entirely clear whether the avoidance behavior that is measured by the Face-Turn AAT concerns fear or general dislike of the stimulus. Third, in order to gain complete experimental control in a neutral setting, ecological validity of a real-life social interaction had to be sacrificed. Fourth, for reasons of convenience and homogeneity of the sample, it was chosen to only include females. The advantage of a homogeneous sample is that gender was not confounding the results and could not reduce the power of this study. However, the drawback is that it remains to be investigated whether the results can be generalized to males: men may show other approach patterns toward the same or opposite gender. Last, this study relied on a non-clinical sample, and research is needed to investigate whether the same patterns appear in patients with SAD.

Concluding, the implicit social behavioral responses of high SAs changed from decreased approach responses under neutral circumstances to increased approach in anticipation of social interaction. How these findings can be interpreted is still open for discussion. The increased approach to social stimuli for the high SAs in anticipation of social interaction may indicate the increased salience of social stimuli or the SAs' implicit desire to engage in social interaction. Future studies need to replicate these findings to shed more light on the interpretation of the increased implicit social approach responses of SAs in anticipation of social interaction.

Acknowledgments Dr. Anne Roefs is thanked for her contributions to the set-up of this study. Anneke van de Gevel, Truus Custers and 
Annie Raven are thanked for conducting the study. In addition, we are grateful to the Behavioural Science Institute of Radboud University Nijmegen, The Netherlands, for financial support and facilities, to Frank Leonardt for programming the AAT, and to the reviewers for helpful comments on an earlier version of the article.

Open Access This article is distributed under the terms of the Creative Commons Attribution Noncommercial License which permits any noncommercial use, distribution, and reproduction in any medium, provided the original author(s) and source are credited.

\section{References}

Alden, L. E. (2001). Interpersonal perspectives on social phobia. In R. W. Crozier \& L. E. Alden (Eds.), International handbook of social anxiety. Concepts, research and interventions relating to the self and shyness (pp. 381-404). Chichester: Wiley.

Alden, L. E., \& Taylor, C. T. (2004). Interpersonal processes in social phobia. Clinical Psychology Review, 24, 857-882.

Alden, L. E., \& Taylor, C. T. (2010). Interpersonal processes in social anxiety disorder. In J. G. Beck (Ed.), Interpersonal processes in the anxiety disorders: Implications for understanding psychopathology and treatment (pp. 125-152). Washington, DC: American Psychological Association.

Alden, L. E., \& Wallace, S. T. (1995). Social phobia and social appraisal in successful and unsuccessful social interactions. Behaviour Research and Therapy, 33, 497-505.

American Psychiatric Association. (2001). Diagnostic and statistical manual of mental disorders text revision (DSM-IV-TR) (4th ed.). Washington, DC: American Psychiatric Association.

Bögels, S. M., Alden, L., Beidel, D. C., Clark, L. A., Pine, D. S., Stein, M. B., et al. (2010). Social anxiety disorder: Questions and answers for the DSM-V. Depression and Anxiety, 27, 168-189.

Boswell, P. C., \& Murray, E. J. (1981). Depression, schizophrenia and social attraction. Journal of Counseling and Clinical Psychology, 49, 641-647.

Cartwright, F., \& Stritzke, W. G. K. (2008). A multidimensional ambivalence model of chocolate craving: Construct validity and associations with chocolate consumption and disordered eating. Eating Behaviors, 9, 1-12.

Chen, M., \& Bargh, J. A. (1999). Consequences of automatic evaluation: Immediate behavioral predispositions to approach or avoid the stimulus. Personality and Social Psychology Bulletin, $25,215-224$

Clark, D. M. (2001). A cognitive perspective on social phobia. In W. R. Crozier \& L. E. Alden (Eds.), International handbook of social anxiety: Concepts, research and interventions relating to the self and shyness (pp. 405-430). New York, NY: Wiley.

Coyne, J. C. (1976). Depression and the response of others. Journal of Abnormal Psychology, 85, 186-193.

Creed, A. T., \& Funder, D. C. (1998). Social anxiety: From the inside and outside. Personality and Individual Differences, 25, 19-33.

Cuming, S., Rapee, R. M., Kemp, N., Abbott, M. J., Peters, L., \& Gaston, J. E. (2009). A self-report measure of subtle avoidance and safety behaviors relevant to social anxiety: Development and psychometric properties. Journal of Anxiety Disorders, 23, 879-883.

Davila, J., \& Beck, J. G. (2002). Is social anxiety associated with impairment in close relationships? A preliminary investigation. Behavior Therapy, 33, 427-446.

Foa, E. B., \& Kozak, M. J. (1985). Treatment of anxiety disorders: Implications for psychopathology. In A. H. Tuma \& J. D. Maser
(Eds.), Anxiety and the anxiety disorders (pp. 421-452). Hillsdale, NJ: Lawrence Erlbaum.

Furukawa, T. A., Chen, J., Watanabe, N., Nakano, Y., Ietsugu, T., Ogawa, S., et al. (2009). Videotaped experiments to drop safety behaviors and self-focused attention for patients with social anxiety disorder: Do they change subjective and objective evaluations of anxiety and performance? Journal of Behavior Therapy and Experimental Psychiatry, 40, 202-210.

Heuer, K., Rinck, M., \& Becker, E. S. (2007). Avoidance of emotional facial expressions in social anxiety: The approachavoidance task. Behaviour Research and Therapy, 45, 29903001 .

Hofmann, S. G. (2007). Cognitive factors that maintain social anxiety disorder: A comprehensive model and its treatment implications. Cognitive Behaviour Therapy, 36, 193-209.

Kashdan, T. B., \& Wenzel, A. (2005). A transactional approach to social anxiety and the genesis of interpersonal closeness: Self, partner, and social context. Behavior Therapy, 36, 335-346.

Kim, E. J. (2005). The effect of the decreased safety behaviors on anxiety and negative thoughts in social phobics. Journal of Anxiety Disorders, 19, 69-86.

Lange, W.-G., Keijsers, G., Becker, E. S., \& Rinck, M. (2008). Social anxiety and evaluation of social crowds: Explicit and implicit measures. Behaviour Research and Therapy, 46, 932-943.

Liebowitz, M. R. (1987). Social phobia. Modern Problems in Pharmacopsychiatry, 22, 141-173.

Mackintosh, B., \& Mathews, A. (2003). Don't look now: Attentional avoidance of emotionally valenced cues. Cognition and Emotion, 17, 623-646.

Mattick, R. P., \& Clarke, J. C. (1998). Development and validation of measures of social phobia scrutiny fear and social interaction anxiety. Behaviour Research and Therapy, 36, 455-470.

McManus, F., Sacadura, C., \& Clark, D. M. (2008). Why social anxiety persists? An experimental investigation of the role of safety behaviours as a maintaining factor. Journal of Behavior Therapy and Experimental Psychiatry, 39, 147-161.

McManus, F., Clark, D. M., Grey, N., Wild, J., Hirsch, C., Fennell, M., et al. (2009). A demonstration of the efficacy of two of the components of cognitive therapy for social phobia. Journal of Anxiety Disorders, 23, 496-503.

Meleshko, K. G., \& Alden, L. E. (1993). Anxiety and self-disclosure: Toward a motivational model. Journal of Personality and Social Psychology, 64, 1000-1009.

Najmi, S., Hindash, A. C., \& Amir, N. (2010). Executive control of attention in individuals with contamination-related obsessivecompulsive symptoms. Depression and Anxiety, 27, 807-812.

Nelson, E. E., Leibenluft, E., McClure, E., \& Pine, D. S. (2005). The social re-orientation of adolescence: A neuroscience perspective on the process and its relation to psychopathology. Psychological Medicine: A Journal of Research in Psychiatry and the Allied Sciences, 35, 163-174.

Papsdorf, M., \& Alden, L. (1998). Mediators of social rejection in social anxiety: Similarity, self-disclosure, and overt signs of anxiety. Journal of Research in Personality, 32, 351-369.

Radloff, L. S. (1977). The CES-D scale: A self-report depression scale for research in the general population. Applied Psychological Measurement, 1, 385-401.

Rapee, R. M., \& Heimberg, R. G. (1997). A cognitive-behavioral model of anxiety in social phobia. Behaviour Research and Therapy, 35, 741-756.

Rinck, M., \& Becker, E. S. (2007). Approach and avoidance in fear of spiders. Journal of Behavior Therapy and Experimental Psychiatry, 38, 105-120.

Rinck, M., Rörtgen, T., Lange, W.-G., Dotsch, R., Wigboldus, D. H. J., \& Becker, E. S. (2010). Social anxiety predicts avoidance 
behaviour in virtual encounters. Cognition and Emotion, 24, 1269-1276.

Roelofs, K., Elzinga, B. M., \& Rotteveel, M. (2005). The effects of stress-induced cortisol responses on approach-avoidance behavior. Psychoneuroendocrinology, 30, 665-677.

Roelofs, K., van Peer, J., Berretty, E., de Jong, P., Spinhoven, P., \& Elzinga, B. M. (2009). Hypothalamus-pituitary-adrenal axis hyper responsiveness is associated with increased social avoidance behavior in social phobia. Biological Psychiatry, 65, 336-343.

Roelofs, K., Putman, P., Schouten, S., Lange, W.-G., Volman, I., \& Rinck, M. (2010). Gaze direction differentially affects avoidance tendencies to happy and angry faces in socially anxious individuals. Behaviour Research and Therapy, 48, 290-294.

Solarz, A. K. (1960). Latency of instrumental responses as a function of compatibility with the meaning of eliciting verbal signs. Journal of Experimental Psychology, 59, 239-245.

Stritzke, W., McEvoy, P., Wheat, L., Dyer, K., \& French, D. (2007). The yin and yang of indulgence and restraint: The ambivalence model of craving. In P. O'Neal (Ed.), Psychology of Motivation (pp. 31-47). Hauppauge, NY: Nova Science.

Taylor, C. T., \& Alden, L. E. (2010). Safety behaviors and judgmental biases in social anxiety disorder. Behaviour Research and Therapy, 48, 226-237.

Van der Ploeg, F. A., Defares, P. B., \& Spielberger, C. D. (1980). Handleiding bij de Zelf Beoordelings Vragenlijst, ZBV. Lisse, The Netherlands: Swets and Zeitlinger.
Voncken, M. J., \& Bögels, S. M. (2008). Social performance deficits in social anxiety disorder: Reality during conversation and biased perception during speech. Journal of Anxiety Disorders, 22, 1384-1392.

Voncken, M. J., \& Dijk, K. F. L. (under review). Socially anxious get a second chance after being disliked at first sight: The role of self-disclosure in the development of likeability in sequential social contact.

Voncken, M. J., Alden, L. E., Bögels, S. M., \& Roelofs, J. (2008). Social rejection in social anxiety disorder: The role of performance deficits, evoked negative emotions and dissimilarity. British Journal of Clinical Psychology, 47, 439-450.

Voncken, M. J., Dijk, C., de Jong, P. J., \& Roelofs, J. (2010). Not selffocused attention but negative beliefs affect poor social performance in social anxiety: An investigation of pathways in the social anxiety-social rejection relationship. Behaviour Research and Therapy, 48, 984-991.

Vrijsen, J. N., Lange, W.-G., Becker, E. S., \& Rinck, M. (2010). Socially anxious individuals lack unintentional mimicry. Behaviour Research and Therapy, 48, 561-564.

Wells, A., Clark, D. M., Salkovskis, P., Ludgate, J., Hackmann, A., \& Gelder, M. (1995). Social phobia: The role of in-situation safety behaviors in maintaining anxiety and negative beliefs. Behavior Therapy, 26, 153-161.

Winer, D. L., Bonner, T. O., Blaney, P. H., \& Murray, E. J. (1981). Depression and social attraction. Mortivation and Emotion, 5, 153-166. 